\title{
On Spectrum Sharing in Cooperative Multiple Access Networks
}

\author{
Amr El-Sherif ${ }^{1}$, Ahmed K. Sadek ${ }^{2}$, and K. J. Ray Liu ${ }^{1}$ \\ ${ }^{1}$ Department of Electrical and Computer Engineering, \\ University of Maryland, College Park, MD 20742, USA. \\ \{aasherif, kjrliu\}@umd.edu \\ ${ }^{2}$ Corporate R\&D Qualcomm Inc. \\ San Diego, CA 92121, USA. \\ asadek@qualcomm.com
}

\begin{abstract}
The coexistence and resource sharing between a primary network utilizing cognitive relays and a secondary network are investigated. Two multiple access protocols are proposed to coordinate idle time slots sharing between the two networks in a TDMA setting. While relays in the primary network are using these free time slots to help users in the primary network forward their unsuccessfully transmitted packets, users of the secondary network use these time slots to transmit their own packets. The diversitymultiplexing sort of tradeoff imposed by this resource sharing problem and optimum resource allocation strategy are studied through the characterization of the maximum stable throughput region of both networks.
\end{abstract}

\section{INTRODUCTION}

The continuous dramatic increase in service demands, capacity and higher performance in communications networks is severely limited by the scarcity of two fundamental resources for communications, namely energy and bandwidth. Researchers are currently focusing on communications and signal processing techniques that can intelligently and efficiently utilize these scarce resources. Among the new techniques that have emerged recently are cooperative communications and cognitive spectrum sharing, both techniques have shown great potential for enhancing the performance of wireless networks and meeting the demands of future wireless applications.

In cooperative communications [1], [2], [3], one or more relays cooperate with a source node to help in forwarding its data to a destination. This can achieve spatial diversity as the data is transmitted via spatially independent channels. In the previous works mentioned above, portion of the channel resources are assigned to the relay for cooperation, which results in some bandwidth efficiency loss. The authors in [4] developed a cooperative multiple-access protocol in which the relay utilizes the empty time slots in a TDMA frame to retransmit the failed transmitted packets of users in the network, thus it does not require allocating part of the channel resources for cooperation. Dynamic spectrum sharing is another promising technique to better utilize the available channel resources [5], [6], [7]. It is well established now that the scarce spectrum is underutilized (spectral holes). One way to reuse it is by introducing a secondary system that opportunistically shares the spectrum of the primary users through the utilization of cognitive radios.

Cooperative communications and spectrum sharing are usually considered as separate problems. In fact, one can consider these two problems as dual problems in the sense that the available unused or under-used channel resources can be utilized to enhance the primary system performance via cooperation, or it can be shared by a secondary system to transmit new information. To study this duality, we investigate the coexistence of a secondary network within a TDMA network employing cognitive relays.
While cooperative and cognitive relays utilize empty time slots in a TDMA frame to retransmit the failed transmitted packets of users in the primary network, secondary users are trying to utilize these empty time slots to transmit their own packets. We propose two different multiple access protocols that coordinate access to channel resources between primary network's relays and secondary users. In the first protocol secondary users are aware of the presence of relays and can sense their transmissions, while in the second protocol both relays and secondary users are competing for free channel resources. For each of these protocols the maximum stable throughput region is characterized and compared with the case where the primary network doesn't employ relays. Moreover, the diversity-multiplexing like tradeoff between the amount of help offered by relays to the primary network and the maximum achievable throughput of the secondary network imposed by resource sharing between relays and secondary users is studied. Our results reveal that although relays are competing for resources with secondary users, both primary and secondary networks exhibit a significant increase in throughput due to cooperation.

\section{Network And Channel Models}

We consider the uplink of a TDMA system as our primary network. The primary network consists of $M$ source terminals numbered $1,2, \ldots, M$, a relay node $r$, and a destination node $d$. In this paper, we will consider the case of a single node $s$ secondary network and two nodes primary network (cf. Fig. 1).

\section{A. Queuing Model}

Each of the two primary nodes, the relay $r$, and the secondary node $s$ has an infinite buffer for storing fixed length packets. The channel is slotted, and a slot duration is equal to a packet duration. The arrival process at $i^{t h}$ node queue $(i \in\{1,2, s\})$ is independent identically distributed (i.i.d) from one slot to another, and the arrival processes are independent from one node to another. The arrival process at the $i^{\text {th }}$ queue is assumed stationary with mean $\lambda_{i}$. Hence, the vector $\boldsymbol{\Lambda}=\left(\lambda_{1}, \lambda_{2}, \lambda_{s}\right)$ denotes the average arrival rates. The arrival process at the relay's queue $i=r$ is analyzed in next section. Primary users access the channel via dividing the channel resources, time in this case, among them, hence, each user is allocated a fraction of the time. Let $\boldsymbol{\Omega}=\left(\omega_{1}, \omega_{2}\right)$ denotes a resource-sharing vector, where $\omega_{i} \geq 0$ is the fraction of time allocated to user $i$. In other words, one can think of $\omega_{i}$ as the probability that a user $i$ is allocated the whole time slot [8]. The set of all feasible resource-sharing vectors is $\digamma=\left\{\boldsymbol{\Omega}=\left(\omega_{1}, \omega_{2}\right) \in \Re^{+2}: \omega_{1}+\omega_{2} \leq 1\right\}$.

The system is called stable for a given arrival rate vector and resource-sharing vector pair $(\Lambda, \Omega)$ if all the queues are stable, 


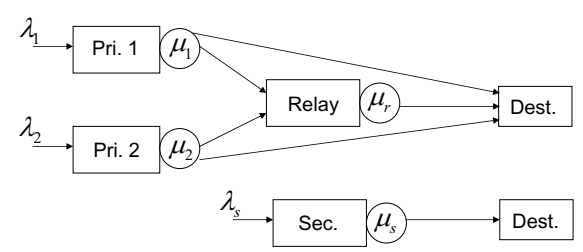

Fig. 1. Network's queuing model

i.e., the primary and secondary nodes and the relay's queues are stable. If any queue is unstable, then the whole system is considered unstable. For an irreducible and aperiodic Markov chain with countable number of states, the chain is stable if and only if there is a positive probability for every queue of being empty, i.e.,

$$
\lim _{t \rightarrow \infty} \operatorname{Pr}\left\{Q_{i}(t)=0\right\}>0 .
$$

(For a rigorous definition of stability under more general scenarios see [9] and [10]). If the arrival and departure processes of a queuing system are strictly stationary, then one can apply Loynes' theorem to check for stability conditions [11]. This theorem states that, if the arrival and departure processes of a queuing system are strictly stationary, and the average arrival rate is less than the average departure rate, then the queue is stable; if the average arrival rate is greater than the average departure rate, then the queue is unstable.

\section{B. Channel Model}

The wireless channel between any two nodes in the network is modeled as Rayleigh flat fading channel with additive white Gaussian noise. The signal received at a receiving node $j$ from a transmitting node $i$ at time $t$ can be modeled as

$$
y_{i j}^{t}=\sqrt{G l_{i j}^{-\gamma}} h_{i j}^{t} x_{i}^{t}+n_{i j}^{t},
$$

where $G$ is the transmitting power, assumed to be the same for all nodes, $l_{i j}$ denotes the distance between the two nodes, $\gamma$ the path loss exponent, $h_{i j}^{t}$ is the channel gain between nodes $i$ and $j$ at time $t$ and is modeled as i.i.d zero mean, circularly symmetric complex gaussian random process with unit variance. The term $x_{i}^{t}$ denotes the transmitted packet with average unit power, and $n_{i j}^{t}$ denote i.i.d additive white Gaussian noise processes with zero mean and variance $N_{0}$. Since the arrivals, the channel gains, and the additive noise processes are all assumed stationary, we can drop the index $t$ without loss of generality.

In this paper, we characterize the success and failure of packet reception by outage events. Outage between two nodes $i$ and $j$ is defined as the event that the received SNR falls below a certain threshold $\beta$

$$
O_{i j} \triangleq\left\{h_{i j}: \frac{\left|h_{i j}\right|^{2} l_{i j}^{-\gamma} G}{N_{o}} \leq \beta\right\} .
$$

If the received SNR is higher than the threshold $\beta$, the receiver is assumed to be able to decode the received message with negligible probability of error. Given the channel model above, the outage probability can be calculated as follows

$$
\operatorname{Pr}\left\{O_{i j}\right\}=1-\exp \left(-\frac{\beta N_{o} l_{i j}^{\gamma}}{G}\right) .
$$

Since we will use frequently the above expression in our subsequent analysis, and for compactness of representation, we will use the following function to denote the success probability,

$$
f_{i j}=\exp \left(-\frac{\beta N_{o} l_{i j}^{\gamma}}{G}\right) .
$$

\section{PRotocols And AnAlysis}

In this work, we are investigating the coexistence of a cooperative relay and a secondary node within the primary TDMA network. Both the relay and secondary node sense the medium for possible opportunities to send their packets. While the secondary node is trying to transmit its own packets, the relay is using idle time slots to help primary nodes, hence, introducing some sort of spatial diversity and increased reliability to the primary network.

First, we describe relay operation. We adopt the protocol presented in [4] as our cognitive cooperative multiple access protocol.

- At the beginning of a time slot, the node transmits the packet at the head of its queue. Due to the broadcast nature of wireless channel, the relay can also receive the transmitted packet with some success probability.

- If the packet is not received correctly by the destination, then a negative acknowledgment (NACK) declaring the packet's failure is fed back from the destination. In this case, if the relay was able to receive the packet correctly then it stores it in its queue waiting for retransmission and sends back an ACK. We assume that the ACK/NACK feedback is immediate and error free.

- The node drops the packet from its queue if it is correctly received by either the destination or the relay.

- At the beginning of each time slot, the relay senses the channel to check if there is a transmission. If the channel is free then the relay transmits the packet at the head of its queue. Here we assume that there is enough guard time at the beginning of each time slot that enables sensing, and that channel sensing is error free.

Next, we propose two different protocols that define channel access rules for the primary network's relay and the secondary node.

\section{A. Protocol $S_{1}$}

In this protocol we assume that the secondary node has the ability to sense the presence of relay transmission (through the presence of long enough guard time at the beginning of a time slot). Moreover, as it is the case with the relay, we assume error free channel sensing at the secondary node. For the relay not to be using all idle time slots, thus inhibiting secondary node access to the channel, the relay will use the channel with some access probability $p_{r}$. Therefore, if the relay's queue is backlogged, and upon the detection of a free time slot the relay will send the packet at the head of its queue with probability $p_{r}$ and leave the slot unused with probability $1-p_{r}$. This will guarantee that the secondary node is assigned at least a fraction $1-p_{r}$ of the free time slots.

Now we are ready to characterize the stability region for protocol $S_{1}$. For the whole system to be stable, all queues 
therein should be stable. Hence, the stability region of the system is the intersection of the stability regions of the two primary nodes' queues, the relay's queue and the secondary node's queue. First, consider the stability region for the system defined by the primary nodes' queues. A primary node succeeds in transmitting a packet if either the destination or the relay receives the packet correctly. Therefore, the success probability of node $i$ can be calculated as

$$
P_{i}=\operatorname{Pr}\left\{\overline{O_{i d}} \bigcap \overline{O_{i r}}\right\}=f_{i d}+f_{i r}-f_{i d} f_{i r},
$$

where $\overline{O_{i j}}$ denotes complement of the event that the channel between user $i$ receiver $j \in(r, d)$ is in outage (i.e., the event that the packet was received correctly). Since for each queue $i$, the queue behaves exactly as in a TDMA system with success probability determined by (6), and from Loynes's theorem, the primary nodes' stability region $\mathcal{R}_{p}\left(S_{1}\right)$ is defined as

$$
\begin{array}{r}
\mathcal{R}_{p}\left(S_{1}\right)=\left\{\left(\lambda_{1}, \lambda_{2}\right) \in R^{+2}: \lambda_{i}<\omega_{i} P_{i}, \forall i \in\{1,2\},\right. \\
\left.\left(\omega_{1}, \omega_{2}\right) \in \digamma\right\} .
\end{array}
$$

Next we consider the stability of the relay's queue $r$. In order to apply Loynes' theorem, we require the arrival and service processes for the relay's queue to be stationary. Let $Q_{r}^{t}$ denote the relay's queue size at time $t$, then its evolution can be modeled as

$$
Q_{r}^{t+1}=\left(Q_{r}^{t}-Y_{r}^{t}\right)^{+}+X_{r}^{t},
$$

where $X_{r}^{t}$ represents the number of arrivals in time slot $t$ and $Y_{r}^{t}$ denotes the possibility of serving a packet at this time slot from relay's queue $\left(Y_{r}^{t}\right.$ takes values $\{0,1\}$ ). Function $(\cdot)^{+}$is defined as $(x)^{+}=\max (x, 0)$. Now we establish the stationarity of the arrival and service processes. If the primary nodes' queues are stable, then by definition the departure processes from both nodes are stationary. A packet departing from a node's queue is stored in the relay's queue (i.e., counted as an arrival) if simultaneously the following two events happen: the node-destination channel is in outage and the node-relay channel is not in outage. Hence, the arrival process to the queue can be modeled as follows

$$
X_{r}^{t}=\sum_{i} \mathbf{1}\left[A_{i}^{t} \bigcap\left\{Q_{i}^{t} \neq 0\right\} \bigcap O_{i d} \bigcap \overline{O_{i r}}\right],
$$

where $\mathbf{1}[\cdot]$ is the indicator function, $A_{i}^{t}$ denotes the event that slot $t$ is assigned to node $i .\left\{Q_{i}^{t} \neq 0\right\}$ denotes the event that node $i$ queue is not empty, i.e., the node has a packet to transmit, and according to Little's theorem [12] it has probability $\lambda_{i} /\left(\omega_{i} P_{i}\right)$, where $P_{i}$ is node $i$ success probability and is defines in (6). The random processes involved in the above expression are all stationary, hence, the arrival process to the relay is stationary. The average arrival rate to the relay's queue can be computed as

$$
\lambda_{r}=E\left[X_{r}^{t}\right]=\sum_{i} \lambda_{i} \frac{\left(1-f_{i d}\right) f_{i r}}{P_{i}} .
$$

Similarly, we establish the stationarity of the service process from the relay's queue. The service process of the relay's queue depends by definition on the empty slots available from primary nodes, and the channel from relay to destination being not in outage. By assuming the nodes' queues to be stable, they offer stationary empty slots (stationary service process) to the relay. Also the channel statistics is stationary, hence, the relay's service process is stationary. The service process of the relay's queue can be modeled as

$$
Y_{r}^{t}=\sum_{i} \mathbf{1}\left[A_{i}^{t} \bigcap\left\{Q_{i}^{t}=0\right\} \bigcap \overline{O_{r d}^{t}} \bigcap U^{t}\right],
$$

where $U^{t}$ is the event that the relay has permission to use the current free time slot to service its queue, which has probability $p_{r}$. The average service rate of the relay can then be determined from the following equation

$$
\mu_{r}=E\left[Y_{r}^{t}\right]=\left(1-\sum_{i} \frac{\lambda_{i}}{P_{i}}\right) f_{r d} p_{r} .
$$

Using Loynes' theorem and equations (11) and (12), the stability region for the relay $\mathcal{R}_{r}\left(S_{1}\right)$ is determined by the condition $\lambda_{r}<$ $\mu_{r}$.

Finally, we consider the stability of the secondary node's queue. The secondary arrival process is stationary with mean $\lambda_{s}$. Secondary node's service process depends on the empty slots available from primary nodes and the relay, and on the channel between secondary node and destination being not in outage. Secondary nodes's service process can then be modeled as

$Y_{s}^{t}=\sum_{i} \mathbf{1}\left[A_{i}^{t} \bigcap\left\{Q_{i}^{t}=0\right\} \bigcap\left\{\overline{Q_{r}^{t} \neq 0 \bigcap U^{t}}\right\} \bigcap \overline{O_{s d}^{t}}\right]$,

where $\overline{\left\{Q_{r}^{t} \neq 0\right\} \cap U^{t}}$ is equivalent to the event that either the relay's queue is empty, or it is backlogged and relay doesn't have permission to access the channel. Given that both primary nodes's queues and relay's queue are stable, then all the events involved in the above expression are stationary, hence, the secondary terminal's service process is stationary, and the average service rate is given by

$\mu_{s}=E\left[Y_{s}^{t}\right]=f_{s d}\left(1-\sum_{i} \frac{\lambda_{i}}{P_{i}}-\frac{1}{f_{r d}} \sum_{i} \lambda_{i} \frac{\left(1-f_{i d}\right) f_{i r}}{P_{i}}\right)$

Using Loynes' theorem, the stability region of the secondary node's queue $\mathcal{R}_{s}\left(S_{1}\right)$ is given by the condition $\lambda_{s}<\mu_{s}$. Finally, the stability region of the whole system is given by the intersection of the three stability regions regions $\mathcal{R}\left(S_{1}\right)=$ $\mathcal{R}_{p}\left(S_{1}\right) \cap \mathcal{R}_{r}\left(S_{1}\right) \cap \mathcal{R}_{s}\left(S_{1}\right)$ which can be easily shown to be equal to $\mathcal{R}\left(S_{1}\right)=\mathcal{R}_{r}\left(S_{1}\right) \cap \mathcal{R}_{s}\left(S_{1}\right)$.

It can be seen from stability conditions and equation (14) that the stability region of the secondary queue does not depend on the fraction $\left(1-p_{r}\right)$ of resources assigned to the secondary node. This result may seem counter-intuitive at first, but careful inspection of the system's queueing model leads to the following interpretation. For a given arrival rate vector $\left(\lambda_{1}, \lambda_{2}\right)$, the relay's average arrival rate $\lambda_{r}$ given by (11) will be constant. Moreover, it is well known that the average departure rate from a stable queue is equal to its average arrival rate. Then given that both the primary nodes' queues and the relay's queue are stable, and as long as the variation in the value of $p_{r}$ does not affect their stability, the average number of free time slots seen by the secondary node will not be affected by the value of $p_{r}$ because the average departure rates from the primary nodes and the relay node will always be $\left(\lambda_{1}, \lambda_{2}\right)$ and $\lambda_{r}$, respectively. 


\section{B. Protocol $S_{2}$}

In this protocol we assume that secondary node cannot sense the presence of relay transmission. To gain access to the channel relay and secondary node have to go through an ALOHA-like contention process. Both relay and secondary node sense the medium for free time slots. When an idle slot is detected the relay and secondary node transmit the packet at the head of their queues with access probability $\alpha_{r}$ for the relay and $\alpha_{s}$ for the secondary node. If a collision between two packets occurs, both packets are lost.

Next, we will characterize the stability region $R\left(S_{2}\right)$ for this system. From the primary nodes point of view, this protocol is equivalent to protocol $S_{1}$. Therefore, the stability region for the primary nodes' queues is given by (7) with success probability $p_{i}$ given by (6). It is noted that the relay and secondary node queues are interacting. In other words, the service process of one queue depend on whether the other other queue is empty or not. Studying the stability conditions for interacting queues is a difficult problem that has been addressed for ALOHA systems [10], [13]. The concept of dominant systems was introduced and employed in [10] to help finding bounds on the stability region of ALOHA with collision channel.

To analyze the stability of the queues in our system, we construct two parallel dominant systems $\left(S_{2}^{1}, S_{2}^{2}\right)$ as follows. For $j \in\{1,2\}$, define $S_{2}^{j}$ as

- Arrivals at each queue in $S_{2}^{j}$ are the same as in $S_{2}$ (the original system).

- Time slots assigned to user $i$ are identical for both $S_{2}$ and $S_{2}^{j}$.

- The outcomes of the "coin tossing" (that determine transmission attempts of the relay and secondary node) in every slot are the same.

- Channel realizations for both systems are identical.

- The noise generated at receiving ends of both systems are identical.

- In $S_{2}^{1}$ the relay continues to transmit dummy packets when its queue is empty, while in $S_{2}^{2}$ the secondary node is transmitting dummy packets when its queue is empty.

The above definition of the dominant system implies that the primary nodes' queues are identical to their counterparts in $S_{2}$ since their departure rates are not affected by the state of the relay and secondary queues. If both the dominant system and the original system started from the same initial queue sizes, then the queues in $S_{2}^{j}$ are always not shorter than those in $S_{2}$. This follows because the probability of a collision as seen from the secondary node will be higher in $S_{2}^{1}$ compared to $S_{2}$ because the relay has always a packet to transmit (possibly dummy). This implies that the secondary node's queue empties faster in $S_{2}$ and therefore the relay's queue will exhibit a lower probability of collision. This same reasoning applies to $S_{2}^{2}$ with the relay and secondary node switching roles in transmitting dummy packets. Consequently, stability conditions for the dominant system are sufficient for the stability of the original system.

Consider the stability conditions for system $S_{2}^{1}$ in which the relay continues to send dummy packets when its queue is empty. The primary nodes are not affected by the dominant system, therefore, their stability condition is defined as in (7). Since the relay has always a packet to transmit (possibly dummy), the secondary queue will have a chance to transmit only if the relay has no permission to transmit, otherwise a collision occurs. Therefore, the secondary node's departure process can be modeled as

$$
Y_{s}^{t}=\sum_{i} \mathbf{1}\left[A_{i}^{t} \bigcap\left\{Q_{i}^{t}=0\right\} \bigcap \overline{A_{r}^{t}} \bigcap A_{s}^{t} \bigcap \overline{O_{s d}^{t}}\right],
$$

where $A_{r}^{t}$ denotes the events that the relay has permission to use slot $t$ to transmit a packet, which has probability $\alpha_{r}$, and $A_{s}^{t}$ denotes the same event for the secondary node and has probability $\alpha_{s}$. Stationarity of the service process follows from stationarity of all events involved in (15). The average departure rate for the secondary node's queue is then given by

$$
\mu_{s}=E\left[Y_{s}^{t}\right]=\left(1-\sum_{i} \frac{\lambda_{i}}{P_{i}}\right)\left(1-\alpha_{r}\right) \alpha_{s} f_{s d} .
$$

The relay's arrival process which is independent from secondary node is defined in (9) and its average arrival rate given by (11). The departure process from the relay under $S_{2}^{1}$ can be modeled as

$$
\begin{array}{r}
Y_{r}^{t}=\sum_{i} \mathbf{1}\left[A_{i}^{t} \bigcap\left\{Q_{i}^{t}=0\right\} \bigcap\left\{\left\{Q_{s}^{t}=0\right\} \bigcup \overline{A_{s}^{t}}\right\}\right. \\
\left.\bigcap A_{r}^{t} \bigcap \overline{O_{r d}^{t}}\right],
\end{array}
$$

where $\left\{Q_{s}^{t}=0\right\} \bigcup \overline{A_{s}^{t}}$ denotes the event that either the secondary node's queue is empty or it doesn't have permission to transmit during current time slot, hence, cannot collide with relay transmission. The average departure rate for the relay's queue is then given by

$$
\mu_{r}=E\left[Y_{r}^{t}\right]=\left(1-\sum_{i} \frac{\lambda_{i}}{P_{i}}\right)\left(1-\frac{\lambda_{s} \alpha_{s}}{\mu_{s}}\right) \alpha_{r} f_{r d} .
$$

Applying Loynes' theorem, the stability region of $S_{2}^{1}$ for a given $\left(\alpha_{r}, \alpha_{s}\right)$ vector is defined by the following condition

$$
\lambda_{r}<\mu_{r}, \text { for } \lambda_{s}<\mu_{s} .
$$

The total stability region $R\left(S_{2}^{1}\right)$ for system $S_{2}^{1}$ is then obtained by finding the union of all stability regions as the parameters $\left(\alpha_{r}, \alpha_{s}\right)$ varies over $[0,1]^{2}$. One method to characterize this union is to solve a constrained optimization problem to find the maximum feasible $\lambda_{s}$ corresponding to each feasible $\left(\lambda_{1}, \lambda_{2}\right)$. Calculation of this union will be omitted for the lack of space.

Similarly, one can show that the stability region $R\left(S_{2}^{2}\right)$ of $S_{2}^{2}$ for a given $\left(\alpha_{r}, \alpha_{s}\right)$ vector is defined by the condition

$$
\lambda_{s}<\mu_{s}, \text { for } \lambda_{r}<\mu_{r} .
$$

where

$$
\begin{array}{r}
\mu_{r}=\left(1-\sum_{i} \frac{\lambda_{i}}{P_{i}}\right)\left(1-\alpha_{s}\right) \alpha_{r} f_{r d}, \\
\mu_{s}=\left(1-\sum_{i} \frac{\lambda_{i}}{P_{i}}\right)\left(1-\frac{\lambda_{r} \alpha_{r}}{\mu_{r}}\right) \alpha_{s} f_{s d} .
\end{array}
$$

Again to characterize the whole stability region $R\left(S_{2}^{2}\right)$, we need to take the union over all possible values of $\left(\alpha_{r}, \alpha_{s}\right)$ in $[0,1]^{2}$.

Finally, the stability region for the original system $S_{2}$ will be given by the intersection $\mathcal{R}\left(S_{2}\right)=\mathcal{R}\left(S_{2}^{1}\right) \cap \mathcal{R}\left(S_{2}^{2}\right)$. 


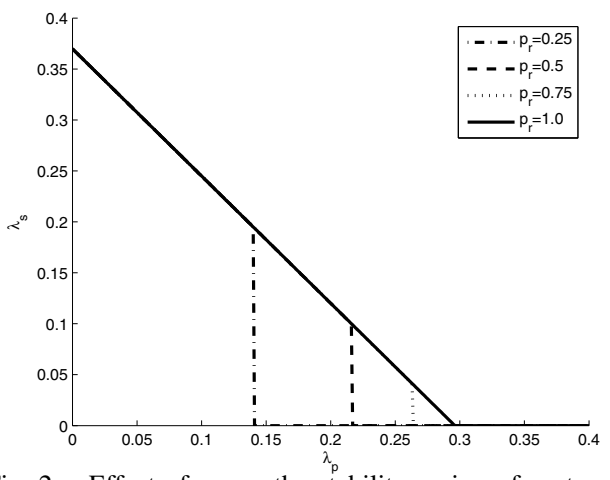

Fig. 2. Effect of $p_{r}$ on the stability region of protocol $S_{1}$

\section{NUMERICAL ReSUltS AND Discussions}

We consider the scenario with only two primary users, which we assume symmetric (i.e., $\lambda_{1}=\lambda_{2}=\lambda_{p}$ ) for ease of illustration. The propagation path loss is taken equal to $\gamma=3.7$ and the SNR threshold $\beta=15 \mathrm{~dB}$. The transmitted signal power is $G=10 \mathrm{~mW}$, and the noise power is $N_{0}=10^{-} 11$. The distances between nodes are, $l_{1 d}=l_{2 d}=100 \mathrm{~m}, l_{1 r}=l_{2 r}=$ $70 \mathrm{~m}, l_{r d}=50 \mathrm{~m}$ and $l_{s d}=110 \mathrm{~m}$.

In Fig. 2 the effect of the parameter $p_{r}$ on the stability region of protocol $S_{1}$ is depicted. It is seen that for a given primary node's arrival rate $\lambda_{p}$ the maximum achievable secondary node's arrival rate $\lambda_{s}$ is independent of $p_{r}$ as was discussed in the previous section. On the other hand, increasing the value of the parameter $p_{r}$ increases the feasible range for $\lambda_{p}$. Based on these results, we conclude that under protocol $S_{1}$ it is always better to give the relay full priority over the secondary node, i.e., the secondary node can only use the channel when both primary nodes' queues and relay queue are empty. This strategy will achieve the maximum stability region.

Fig. 3 compares between protocol $S_{1}$, protocol $S_{2}$ where the relay and secondary node are contending for free time slots and between the case where the primary network doesn't have a relay. First, protocol $S_{1}$ achieves a significant improvement in stability compared to the no relay case. For a given $\lambda_{p}$ we can see more that $100 \%$ increase in the achievable throughput by the secondary user for $\lambda_{p}>0.15$. Although the relay has the priority, and secondary node has to wait till all other queues are empty before transmitting, the presence of the relay helps the primary nodes free their queues at a faster rate leaving more free time slots for the secondary node, which explains the increased throughput. Next, considering the performance of protocol $S_{2}$, it is noted that its stability region is completely below the stability region of $S_{1}$, this result is expected since $S_{1}$ doesn't suffer from collisions between relay and secondary transmissions. Furthermore, these collisions interpret the fact that the no relay case performs better than $S_{2}$ from the point of view of stable throughput; the performance loss due to collisions exceeds the benefits of diversity offered by the relay. Only at high values of $\lambda_{p}$ where protocol $S_{2}$ has a slightly better performance because the effect of relay's help overcomes the degradation due to collisions.

\section{CONCLUSIONS}

In this paper, we proposed two multiple access protocols that enable resource sharing between cooperative relays in a

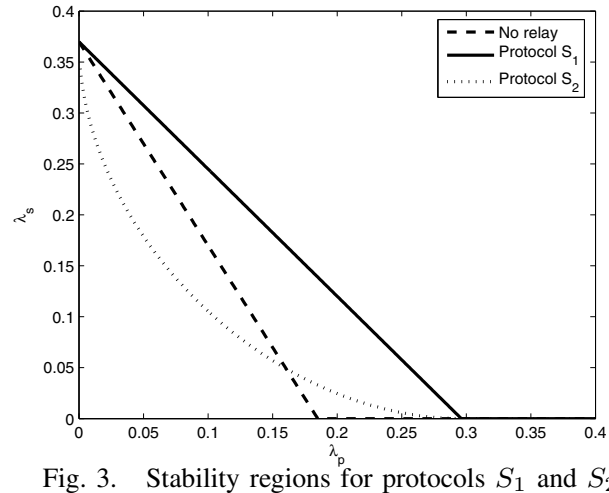

TDMA network and a secondary network. Stability regions of both protocols were characterized. Analysis and numerical results revealed that it is better, from the viewpoint of maximum stable throughput, that relays have priority over secondary nodes accessing free network resources. This will help primary nodes empty their queues at a faster rate, therefore, offering more free time slots for the secondary nodes to utilize, which results in more than $100 \%$ increase in secondary nodes' throughput.

\section{REFERENCES}

[1] J. N. Laneman, D. N. C. Tse, , and G. W. Wornell, "Cooperative diversity in wireless networks: efficient protocols and outage behavior," IEEE Trans. Inform. Theory, vol. 50, pp. 3062-3080, Dec. 2004.

[2] A. K. Sadek, W. Su, and K. J. R. Liu, "Multinode cooperative communications in wireless networks," IEEE Trans. on Signal Processing, vol. 55, no. 1, pp. 341-355, Jan. 2007.

[3] G. Kramer, M. Gastpar, and P. Gupta, "Cooperative strategies and capacity theorems for relay networks," IEEE Trans. on Inform. Theory, Dec. 2005.

[4] A. K. Sadek, K. J. R. Liu, and A. Epheremides, "Cognitive multiple access via cooperation: Protocol design and performance analysis," IEEE Trans. on Information Theory, vol. 53, no. 10, pp. 3677-3696, Oct. 2007.

[5] H. Zheng and C. Peng, "Collaboration and fairness in opportunistic spectrum access," in Proc. of IEEE Internation Conference on Communications (ICC), May 2005.

[6] L. Cao and H. Zheng, "Distributed spectrum allocation via local bargaining," in Proc. of IEEE Conference on Senson and Ad Hoc Communications and networks (SECON), Santa Clara, CA, Sep. 2005.

[7] J. Huang, R. A. Berry, and M. L. Honig, "Auction-based spectrum sharing," ACM/Springer Mobile Networks and Applications Journal (MONET), vol. 11, no. 3, pp. 405-418, Jun. 2006.

[8] M. Kobayashi, G. Caire, and D. Gesbert, "Impact of multiple transmit antennas in a queued sdms/tdma downlink," in Proc. of 6th IEEE Workshop on Signal Processing Advances in Wireless Communications (SPAWC), New York, NY, June 2005.

[9] W. Szpankowski, "Stability conditions for some multiqueue distributed system: Buffered random access systems," Adv. Appl. Probab., vol. 26, pp. 498-515, 1994.

[10] R. Rao and A. Ephremides, "On the stability of interacting queues in a multi-access system," IEEE Trans. on Inform. Theory, vol. 34, pp. 918-930, Sept. 1988.

[11] R. M. Loynes, "The stability of a queue with non-independent interarrival and service times," Proc. Cambridge Philos. Soc., pp. 497-520, 1962.

[12] D. Bertsekas and R. Gallager, Data Networks, Prentice Hall, 2nd edition edition, 1992.

[13] V. Naware, G. Mergen, and L. Tong, "Stability and delay of finite-user slotted aloha with multipacket reception," IEEE Trans. on Inform. Theory, vol. 51, no. 7, pp. 2636-2656, Jul. 2005. 